\title{
Study on the Baseline Factors and Platelet Indices That Predict Outcome of Acute Ischemic Stroke Patients after Thrombolytic Therapy
}

\author{
Yiqin Yao $^{a} \quad$ Xuejin Cao $^{b}$ Rongcheng Zou ${ }^{a}$ Hongbo Wen $^{a}$ Shiyao Zhang ${ }^{b}$ \\ Hui Xu ${ }^{b}$ Xiaoying Guo ${ }^{b}$ Yijing Guo ${ }^{a}$ b
}

aDepartment of Neurology, Nanjing Lishui People's Hospital, ZhongDa Hospital Lishui Branch, Southeast University, Nanjing, China; ${ }^{b}$ Department of Neurology, Affiliated ZhongDa Hospital of Southeast University, Medical School of Southeast University, Nanjing, China

\section{Keywords}

Acute ischemic stroke $\cdot$ Thrombolytic therapy $\cdot$ Functional outcome $\cdot$ Platelet indices · Prognosis

\begin{abstract}
Background: The aim of the study was to investigate the baseline characters that influence 3-month clinical outcomes in patients with acute ischemic stroke (AIS) after thrombolytic therapy. Methods: We consecutively enrolled 241 AIS patients who are treated with thrombolytic therapy with recombinant tissue plasminogen activator. Baseline characters were measured on admission including the $\mathrm{Na}$ tional Institutes of Health Stroke Scale (NIHSS), Trial of Org 10172 in Acute Stroke Treatment (TOAST), risk factors, platelet indices, and lipid parameters. The subjects were divided into good or poor functional outcomes based on modified Rankin Scale at 3 months. The multivariate logistic regression was performed to explore the association between baseline factors and outcomes. Pearson correlation was used to investigate whether linear associations existed between platelet indices in different outcomes. Results: Multivariate logistic regression analysis showed that the NIHSS, TOAST classification, diabetes, mean platelet volume (MPV) are important factors for predicting clinical outcomes after 3 months in AIS patients. We found a correlation between el-
\end{abstract}

evated MPV and worse outcome at 3 months, particularly in large-artery atherosclerosis stroke patients. MPV and platelet count are negative correlated $(r=-0.375, p=0.000)$. MPV and platelet-to-lymphocyte ratio (PLR) $(r=0.83, p=0.000)$, MPV and platelet distribution width (PDW) $(r=0.820, p=$ 0.000 ) both have highly positive linear correlations in patients with good outcome. Conclusions: Overall, lower NIHSS and MPV levels on admission were predictors of good functional outcomes in patients with AIS after undergoing thrombolytic therapy. The correlations between MPV, PDW, and PLR may be helpful to evaluate prognosis in stroke patients and deserve further exploration.

(C) 2021 The Author(s)

Published by S. Karger AG, Basel

\section{Introduction}

Acute ischemic stroke (AIS) occurs mainly due to thrombotic and embolic mechanisms. Hypertension, diabetes mellitus (DM), hyperlipidemia, and abnormal platelet function are among the most important medical pre-conditions and risk factors for ischemic stroke $[1,2]$. Although the usage of recombinant tissue plasminogen

Yiqin Yao and Xuejin Cao contributed equally to this work and should be considered co-first authors. karger@karger.com www.karger.com/ced

Karger $\stackrel{\text { ' }}{5}$

BOPEN ACCESS
(C) 2021 The Author(s)

Published by S. Karger AG, Basel

This is an Open Access article licensed under the Creative Commons Attribution-NonCommercial-4.0 International License (CC BY-NC) (http://www.karger.com/Services/OpenAccessLicense), applicable to the online version of the article only. Usage and distribution for commercial purposes requires written permission.
Correspondence to:

Yijing Guo, guoyijingseu@ 126.com 
activator (rt-PA) has greatly improved the clinical outcome for some patients with AIS, the therapeutic effect of rt-PA by intravenous thrombolysis (IVT) remains different $[3,4]$. The unfavorable functional outcome is determined by various factors, such as older age, higher National Institute of Health Stroke Scale (NIHSS) score, location of the occlusion, underlying vascular conditions, time of reperfusion, and combination of diseases [5]. For these patients, it would be valuable to identify a readily available prognostic biomarker on admission for evaluating the therapeutic strategies on functional outcomes.

In the occurrence of atherosclerosis and thrombosis that may initiate ischemic stroke, a large number of platelets are activated; platelet aggregation and adhesion participate in the pathological process [6]. Clinical platelet indices contain mean platelet volume (MPV), platelet count (PC), platelet distribution width (PDW), and plateletcrit (PCT); they have been investigated in various diseases. Larger platelets are more metabolically and functionally active [7]. Increased MPV is an important marker of platelet activation, with high aggregation activity for containing more granule secretions, thromboxane synthesis, and adhesion molecules [8]. Specifically, some studies have showed that an elevated MPV and MPV/PC ratio were associated with poor prognosis in patients with AIS [9]. Besides, the available data regarding the relationships between other platelet indices (e.g., MPV and PDW) in prognostic role of the outcome in AIS patients treated with rt-PA are lacking. This study aimed to analyze the factors that influence the functional outcome of patients with AIS after IVT and further explored the relationships between platelet indices for prognostic judgment in these patients.

\section{Methods}

\section{Study Population}

This prospective study includes 241 patients who were admitted to the hospital (Nanjing Lishui People's Hospital and Affiliated Zhongda Hospital of Southeast University) within $4.5 \mathrm{~h}$ after the onset of AIS and received IVT between July 2017 and August 2020. Ethical approval was obtained from the Local Ethics Committee and conducted according to the principles of the Declaration of Helsinki.

AIS was diagnosed on the basis of criteria and the diagnosis was confirmed by using either brain computerized tomography or magnetic resonance imaging (T2-FLAIR, diffusion-weighted imaging (T1). Stroke subtype among the patients was classified according to Trial of Org 10172 in Acute Stroke Treatment (TOAST) classification [10]. The enrolled patients received intravenous rtPA $(0.9 \mathrm{mg} / \mathrm{kg})$ therapy. Patients were excluded if they went to mechanical thrombectomy after IV rt-PA. All the patients received the appropriate rehabilitation guidance after the treatment.

\section{Data Collection}

Baseline information of demographics, medical history, blood pressure, onset-to-treatment time, NIHSS before thrombolysis was routinely collected at admission. All patients underwent blood sampling immediately after admission and before IVT. The blood samples were anticoagulated with ethylenediaminetetraacetic. The platelet-related parameters, including MPV, PDW, PC, PCT, and platelet-to-lymphocyte ratio (PLR) were determined using automated hematology analyzer (XS-800i; Sysmex, JP). MPV was measured in units of $\mathrm{fL}$ (normal range, 7.5-11.5 fL). The first overnight fasting venous blood samples were collected for detection of blood glucose and serum lipid containing total cholesterol, triglycerides (TGs), high-density lipoprotein, lowdensity lipoprotein. Computerized tomography scans were performed immediately at admission and $24 \mathrm{~h}$ after rt-PA or earlier in case of neurological deterioration to rule out intracranial hemorrhage. Magnetic resonance imaging scans were completed as long as condition permits. Functional outcomes were evaluated using the modified Rankin scale (mRS) score at 3 months after stroke. We defined mRS 0-2 as a good outcome and mRS 3-6 as a poor outcome.

\section{Statistical Analyses}

Statistical analyses were performed using SPSS 22.0 software. Continuous variables are reported as the means with standard deviations or medians with interquartile ranges. Categorical variables are reported as percentages. Statistical analyses were performed using Student's $t$ tests or the Kruskal-Wallis test for continuous data, and the $\chi^{2}$ test or Fisher's exact test for categorical data. We compared baseline characteristics between the poor and good outcome groups and employed the multivariate logistic regression model to evaluate the baseline factors that predict poor functional outcomes at 3 months. Correlations between platelet indices were evaluated using Pearson correlation test. Statistical significance was set at $p$ value $<0.05$ for all analyses.

\section{Results}

\section{Baseline Characteristics of the Good/Poor Outcome Groups}

A total of 241 patients with first-ever ischemic stroke were included in this study. Table 1 summarizes the characteristics of the patients in both groups: good outcome (mRS score $0-2$ ) and poor outcome (mRS score 3-6). Patients with favorable outcome were less likely to have a history of diabetes $(p=0.007, p<0.01)$ and atrial fibrillation (AF) $(p=0.000, p<0.001)$. Baseline NIHSS score and MPV was significantly higher in patients with poor outcome (both $p=0.000, p<0.001$ ). The good outcome group had smaller possibilities of a prior stroke $(p=0.003$, $p<0.01)$, hemorrhagic transformation (HT) $(p=0.000$, $p<0.001)$, and lower fasting blood glucose levels $(p=$ $0.011, p<0.05)$. 
Table 1. Baseline characteristics in AIS patients with good/poor 3-month outcomes

\begin{tabular}{|c|c|c|c|}
\hline Characteristics & Good, mRS 0-2 $(n=148)$ & Poor, mRS 3-6 $(n=93)$ & $p$ value \\
\hline Age, years, mean $\pm S D$ & $66.6 \pm 0.9$ & $70.1 \pm 1.3$ & 0.023 \\
\hline Male, $n(\%)$ & $99(63.9)$ & $57(61.3)$ & 0.376 \\
\hline Diabetes, $n(\%)$ & $43(29.1)$ & $43(46.2)$ & 0.007 \\
\hline Smoking, $n(\%)$ & $62(41.9)$ & $34(36.6)$ & 0.410 \\
\hline $\mathrm{AF}, n(\%)$ & $31(20.9)$ & $40(43.0)$ & 0.000 \\
\hline Coronary artery disease, $n(\%)$ & $11(7.4)$ & $12(12.9)$ & 0.159 \\
\hline Prior stroke, $n(\%)$ & $14(9.5)$ & $22(23.7)$ & 0.003 \\
\hline Systolic BP, mm Hg, mean \pm SD & $160.5 \pm 25.5$ & $163.6 \pm 27.3$ & 0.374 \\
\hline Baseline NIHSS score, median (IQR) & $4(4)$ & $12(12)$ & 0.000 \\
\hline $\mathrm{OTT}$, min, mean $\pm \mathrm{SD}$ & $164.7 \pm 52.1$ & $171.1 \pm 46.3$ & 0.332 \\
\hline \multicolumn{4}{|l|}{ TOAST, $n(\%)$} \\
\hline LAA & $42(28.4)$ & $48(49.5)$ & 0.001 \\
\hline CE & $22(14.9)$ & $34(36.6)$ & 0.000 \\
\hline SAO & $84(56.8)$ & $13(14.0)$ & 0.000 \\
\hline Hemorrhagic transformation, $n(\%)$ & $7(4.7)$ & $22(23.7)$ & 0.000 \\
\hline $\mathrm{FBG}, \mathrm{mmol} / \mathrm{L}$, mean $\pm \mathrm{SD}$ & $6.7 \pm 2.2$ & $7.5 \pm 3.1$ & 0.011 \\
\hline \multicolumn{4}{|l|}{ Lipid parameters, $\mathrm{mmol} / \mathrm{L}$, mean $\pm \mathrm{SD}$} \\
\hline $\mathrm{TC}$ & $4.4 \pm 1.0$ & $6.0 \pm 15.7$ & 0.216 \\
\hline TG & $1.7 \pm 1.3$ & $1.4 \pm 0.8$ & 0.071 \\
\hline HDL & $1.3 \pm 0.4$ & $1.3 \pm 1.2$ & 0.454 \\
\hline LDL & $2.5 \pm 0.9$ & $2.7 \pm 1.7$ & 0.173 \\
\hline \multicolumn{4}{|l|}{ Platelet indices } \\
\hline $\mathrm{PC}, 10^{9} / \mathrm{L}$, mean $\pm \mathrm{SD}$ & $169.8 \pm 49.5$ & $180.3 \pm 58.1$ & 0.136 \\
\hline $\mathrm{PCT}, \%$, mean $\pm \mathrm{SD}$ & $0.19 \pm 0.05$ & $0.20 \pm 0.63$ & 0.220 \\
\hline$M P V, f L$, mean $\pm S D$ & $11.4 \pm 1.2$ & $12.4 \pm 1.2$ & 0.000 \\
\hline $\mathrm{PDW}, \mathrm{fL}$, mean $\pm \mathrm{SD}$ & $15.3 \pm 3.1$ & $14.6 \pm 3.0$ & 0.075 \\
\hline PLR, \%, mean \pm SD & $37.8 \pm 9.3$ & $36.1 \pm 9.0$ & 0.153 \\
\hline
\end{tabular}

mRS, modified Rankin Scale; SD, standard deviation; IQR, interquartile range; NIHSS, National Institutes of Health Stroke Scale; LAA, large-artery atherosclerosis; CE, cardioembolic; SAO, small-artery occlusion; HDL, high-density lipoprotein; LDL, low-density lipoprotein; FBG, fasting blood glucose; PC, platelet count; PCT, plateletcrit; MPV, mean platelet volume; PDW, platelet distribution width; PLR, platelet-to-lymphocyte ratio; AIS, acute ischemic stroke; TC, total cholesterol; TG, triglyceride; $\mathrm{AF}$, atrial fibrillation; $\mathrm{OTT}$, onset-to-treatment time; $\mathrm{BP}$, blood pressure.

The Predictive Values of Baseline Factors in Poor Outcome of AIS following rt-PA by Multivariate Logistic Regression Analysis

Table 2 shows the multivariate logistic regression analysis for the significant measures that play crucial roles in prognostic judgment. NIHSS, TOAST, MPV, DM, PLR, and TG were considered as independent predictors for stroke outcome. The overall correct predicted value of the logistic regression model was $86.3 \%$ (Table 3 ). Patients with higher MPV (OR 8.732, $p<0.001$ ), lower PLR (OR 0.770, $p<0.001$ ), and TG (OR $0.572, p<0.05$ ) were probably become dependent in daily life at 3 months after stroke. The increased NIHSS score (OR 1.271, $p<0.001$ ) and comorbidities of DM (OR 0.192, $p<0.001$ ) indicated poor outcome. The TOAST classification denotes 5 subtypes of ischemic stroke: (1) large-artery atherosclerosis (LAA); (2) car- dioembolism (CE); (3) small-artery occlusion (SAO); (4) stroke of other determined cause; and (5) stroke of undetermined cause. There are 3 types of TOAST for patients included in this study, TOAST (1): SAO; TOAST (2): LAA; TOAST (3): CE. The model used TOAST (3) as the reference level, $\mathrm{CE}$ type contributed to the different prognosis $(p=0.004, p<0.005)$. Compared with CE, patients with SAO were more likely to have a good outcome $(p=0.013$, $p<0.05$ ), while LAA had no significant effect on the prognosis $(p=0.689)$ with reference to CE. Overall, the TOAST classification would influence the prognosis.

Figure 1 demonstrates the correlation between important baseline factors and the 3-month outcomes. It demonstrated that the more severe the initial symptoms were, the worse the outcomes probably would be. Most of the patients without DM and with SAO have good 
Table 2. Multivariate logistic regression model* of predictors to poor outcome

\begin{tabular}{lllllll}
\hline Characteristic & $\beta$ & SE & $p$ value & OR & \multicolumn{2}{l}{$95 \% \mathrm{Cl}$} \\
\cline { 4 - 7 } & & & & & lower & upper \\
\hline MPV & 2.167 & 0.413 & 0.000 & 8.732 & 3.884 & 19.632 \\
TGs & -0.558 & 0.256 & 0.029 & 0.572 & 0.347 & 0.945 \\
PLR & -0.261 & 0.054 & 0.000 & 0.770 & 0.693 & 0.856 \\
NIHSS & 0.240 & 0.043 & 0.000 & 1.271 & 1.169 & 1.382 \\
Diabetes (1) & 1.651 & 0.472 & 0.000 & 0.192 & 0.076 & 0.483 \\
TOAST (3) & & & 0.004 & & & 0.723 \\
TOAST (1) & -1.530 & 0.615 & 0.013 & 0.217 & 0.065 & 3.758 \\
TOAST (2) & 0.224 & 0.561 & 0.689 & 1.252 & 0.417 & 3 \\
\hline
\end{tabular}

Poor outcome, 3-month mRS score from 3 to $6 ; \beta$, regression coefficients; SE, standard error; OR, odds ratio; $\mathrm{Cl}$, confidence interval; MPV, mean platelet volume; PLR, platelet-to-lymphocyte ratio; NIHSS, National Institutes of Health Stroke Scale; TGs, triglycerides; CE, cardioembolic; SAO, small-artery occlusion; LAA, large-artery atherosclerosis; AF, atrial fibrillation; FBG, fasting blood glucose; mRS, modified Rankin Scale. TOAST, CE (as control); TOAST (1), SAO; TOAST (2), LAA. * NIHSS, TOAST classification, age, gender, diabetes, AF, prior stroke, FBG, platelet volume indices, lipid parameters (Table 1) were included in the multivariate analysis as the independent variables.

Table 3. Correct predicted percentage of the multivariate logistic regression model

\begin{tabular}{llll}
\hline Observed value & \multicolumn{2}{l}{ Predicted value } & \multirow{2}{l}{$\begin{array}{l}\text { Correct } \\
\text { percentage, \% }\end{array}$} \\
\cline { 2 - 3 } & good & poor & \\
\hline Good & 137 & 11 & 92.6 \\
Poor & 22 & 71 & 76.3 \\
Overall percentage & & & 86.3 \\
\hline
\end{tabular}

outcomes. Moreover, we analyzed the distributions of some categorical variables, and found CE subtype was approximately equal to AF (Fig. 2a). In other words, the effect of AF was included by TOAST. The NIHSS score was different between HT group and no-HT group $(p<$ 0.001 ) (Fig. 2b), so the effect of HT could be overlapped partly by NIHSS.

For MPV was the predictor that contributed the most to the outcome (OR 8.732, $p<0.001$ ), it was further investigated. In general, the elevated MPV contributed to worse outcomes at 3 months, particularly in LAA stroke patients (Fig. 1e).

\section{Correlation Analyses of Platelet Indices}

The positive correlation between PC and PCT was almost identical in the whole subjects (Fig. 3a). For it was MPV that has important effect on the functional results, we focused on the relationships between MPV, PDW, and PLR in different outcomes.
Besides MPV and PC are negatively correlated $(r=$ $-0.375, p=0.000)$, MPV and PLR $(r=0.83, p=0.000)$, MPV and PDW $(r=0.820, p=0.000)$ had highly positive linear correlations in patients with good outcome. In the group of poor outcome, MPV and PC are not correlated $(r=-0.068, p=0.259)$, but MPV and PLR $(r=0.285, p=$ $0.003)$ and MPV and PDW $(r=0.285, p=0.003)$ still positively correlated. The PLR and PDW were related in both the good outcome group $(r=0.924, p=0.000)$ and poor outcome group $(r=0.943, p=0.000)$ (Fig. 3). In conclusion, patients with poor outcome at 3 months had lower correlation coefficient between MPV and PDW and MPV and PLR than those who returned to independence.

\section{Discussion}

The study found that NIHSS, TOAST, DM, and MPV were important predictors for functional outcomes at 3 months in AIS patients after undergoing thrombolytic therapy. Lower NIHSS and MPV levels upon admission predicted good outcomes. MPV and PLR and MPV and PDW both have highly positive linear correlations in patients with good outcome. The correlations between MPV, PDW, and PLR may be helpful to evaluate prognosis in stroke patients.

The TOAST criteria are a widely used classification scheme of ischemic stroke subtypes. A prospective study reported that subtype was a significant predictor of survival in stroke patients, and the highest 2-year survival was found in 


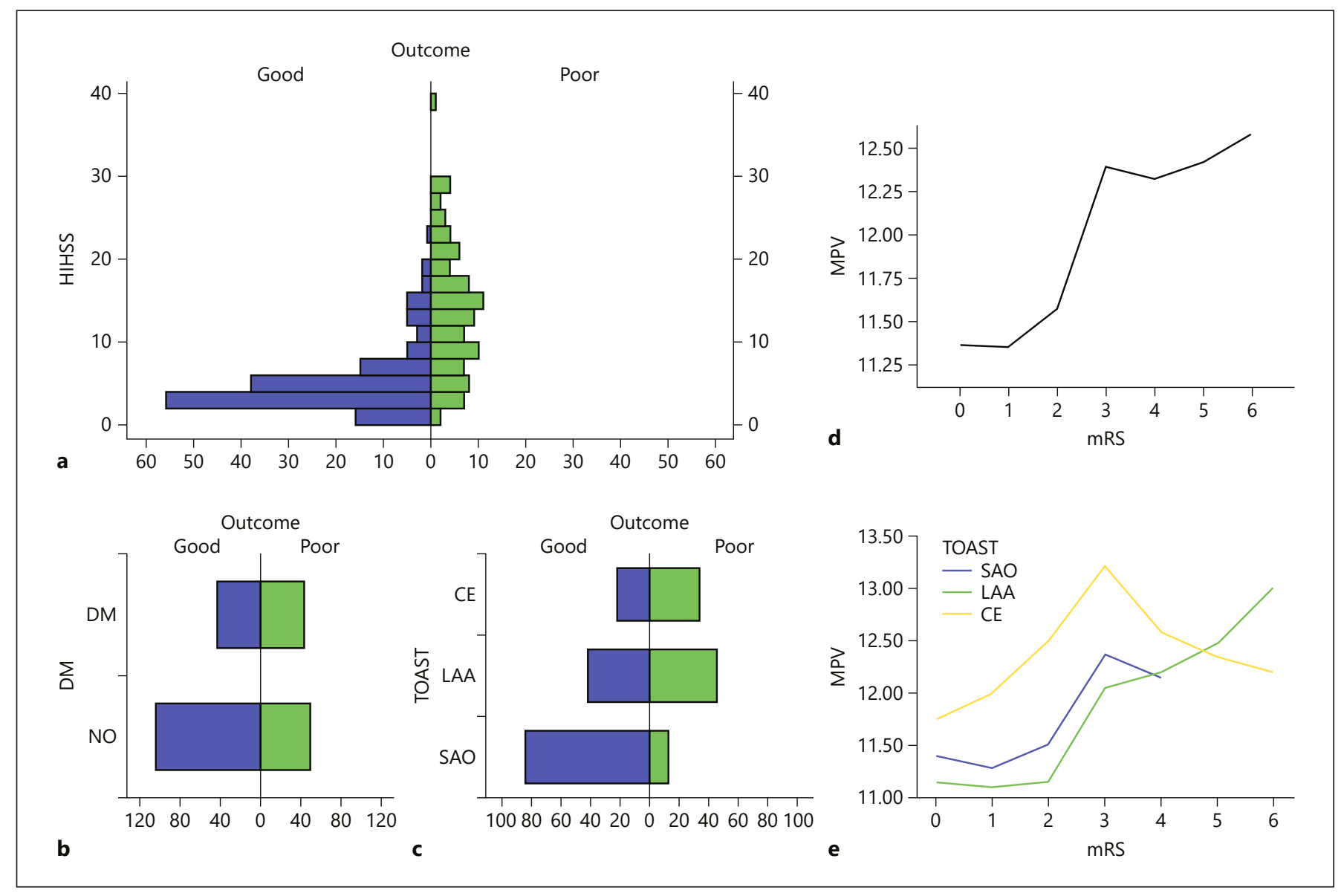

Fig. 1. Illustration of the distributions of predictive factors in the different outcomes. Counts of patients in the 2 outcome groups with NIHSS score (a), DM (b) and TOAST (c) on admission. d, e Correlation of baseline MPV and $\mathrm{mRS}$ at 3 months simply shown in line charts. The line color in (e) indicates different TOAST types. SAO, small-artery occlusion; LAA, large-artery atherosclerosis; CE, cardioembolic; NIHSS, National Institutes of Health Stroke Scale; DM, diabetes mellitus; MPV, mean platelet volume; mRS, modified Rankin Scale.

SAO [11]. SAO are usually the result of occlusion of small penetrating arteries, and our study suggested that most of these patients also develop good prognosis in 3 months.

The MPV level was positively correlated with $\mathrm{mRS}$ in LAA stroke and patients with increased MPV were more likely to develop poor outcomes (Fig. 1e). LAA patients usually have occlusion of a major brain artery or branch cortical artery, presumably due to atherosclerosis [12]. Platelets play a crucial role in atherosclerosis and a cross-link between inflammation and thrombosis $[13,14]$. Platelet activation induces the release of chemokines, which mediate the recruitment of leukocytes (neutrophils, monocytes, or lymphocytes) into the vascular wall, favoring atherosclerotic lesions and atherothrombosis $[15,16]$. Lymphocytes are important subtype of the leukocyte family, and the low lymphocyte count is associated with increased cardiovascular disease
[17]. The clinical significance of PLR has been reported in diseases including coronary artery disease and carotid arterial stenosis. Also, an increased PLR is also detected to be an independent predictor of stroke [18]. Under acute ischemic conditions, increased MPV is linked to more active platelets with higher avidity to aggregate [19]. And increased turnover of platelets causes the release of young, more active, and larger platelets from bone marrow [20].

It could be concluded that over-activation of platelets is an important pathogenic factor and MPV is a predictor of outcomes in patients with LAA stroke. Atherosclerotic arteries causes the platelets to be more easily aggregated and activated, the activation of platelets and inflammation have a negative effect on the blood vessels in turn, leading to a vicious circle. In this case, the larger the MPV, the more serious was the interaction between platelet activation, throm- 


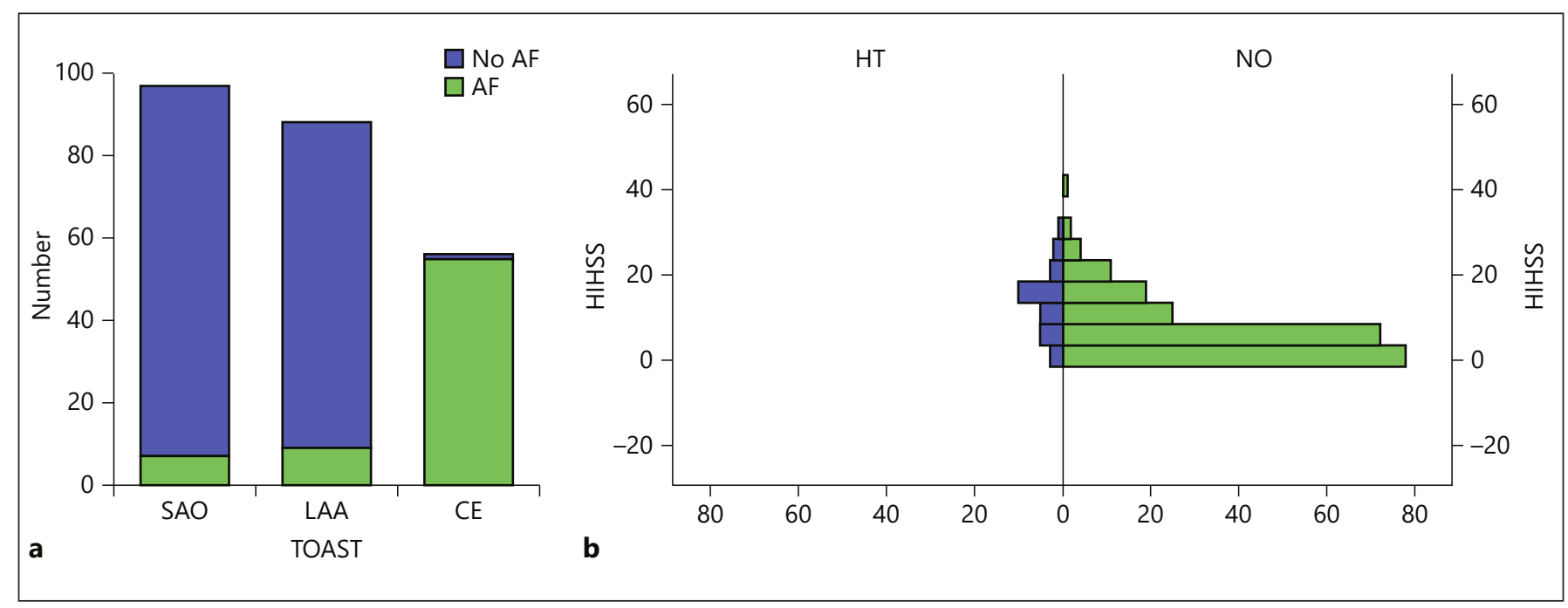

Fig. 2. Distributions of the categorical variables in patients on admission. a Numbers of patients with or without AF in different TOAST types. b Numbers of patients with or without HT in different NIHSS scores. AF, atrial fibrillation; HT, hemorrhagic transformation; NIHSS, National Institutes of Health Stroke Scale; AF, atrial fibrillation; SAO, small-artery occlusion; LAA, large-artery atherosclerosis; CE, cardioembolic.

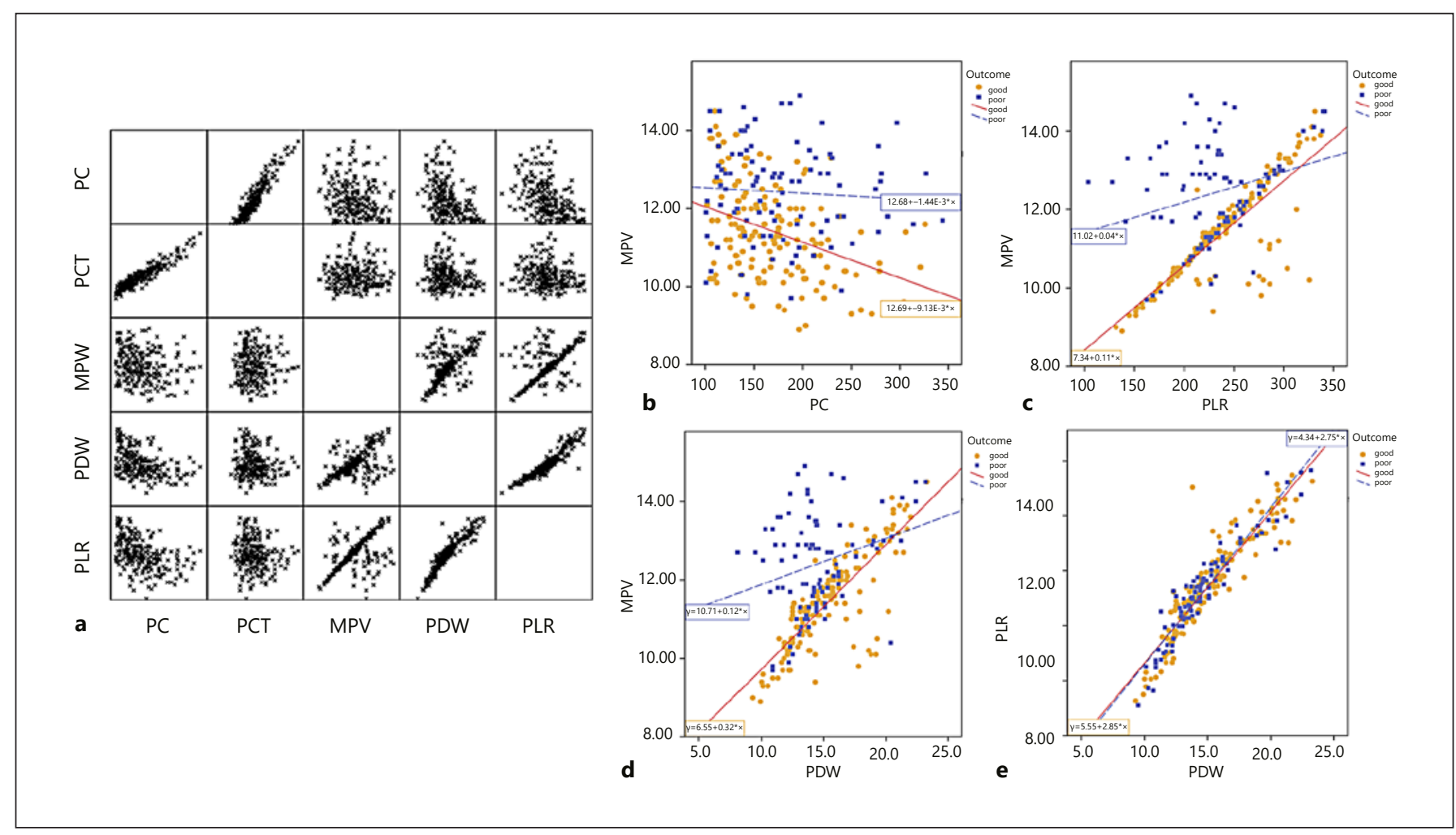

Fig. 3. Correlation analysis between platelet volume indices according to different outcomes. a Matrix of correlation between platelet volume indices demonstrated by scatterplot. $\mathbf{b}$ Linear correlation between MPV and PC. c-e Linear correlation between MPV, PLR, and PDW. PC, platelet count; MPV, mean platelet volume; PDW, platelet distribution width; PLR, platelet-to-lymphocyte ratio; PCT, plateletcrit. 
bosis, and inflammation. In addition of the poor vascular function and condition, the prognosis is usually worse in these patients. Platelet activation and inflammation are also pathology mechanisms in diabetes [21]. The risk factors of atherosclerosis include diabetes, hypertension, hyperlipidemia, smoking, and inflammation. The patients with diabetes had more severe disease situation that they probably had poor outcomes after stroke.

MPV is a marker of platelet activity. In the process of thrombus formation, too large activated platelets may be an indicator of structural and functional disorder. So the MPV and PDW ratio could be out of a specific range when the homeostasis is disturbed. The leukocyte-platelet aggregates have been indicated in research of ischemic events, which acted as a marker of activated platelets and associated with inflammation and thrombus $[15,22,23]$. Increased formation of leukocyte-platelet aggregates after ischemic stroke and reperfusion may aggravate the recruitment of leukocytes. Therefore, the MPV and PLR also remain balanced if the process is in a relatively tolerable physiological range. The correlation coefficient between MPV and PLR in patients with poor outcome was smaller than patients with good outcome at 3 months, which reflect that the interaction of platelets and leukocyte were out of proportion.

Some studies showed that decreased PC, elevated MPV, and MPV/PC ratio were associated with poor prognosis in stroke patients $[7,9,24]$. We analyzed the correlation of MPV and PC in order to verify this. According to the results, the correlation of MPV and PC was different in the 2 outcome groups. However, the scattered points in the graph of MPV/PDW and MPV/PLR were distributed in a way that can better distinguish the 2 groups (Fig. 3b-d) than MPV/PC. Among the platelet indices, MPV was the most important prognostic indicator of outcome in AIS patients. In patients with poor outcomes, the lower correlation between MPV, PDW, and PLR might reflect some changes in specific characteristics of the platelet and the unbalance of platelets and leukocytes within the blood stream. The exact mechanism still remains unclear.

It was difficult to explore the relationships between all the variables completely. Our research was an attempt to find out some potential correlations in these data of stroke patients. We hope to have a larger sample size and more detailed analysis method to validate the findings. The study pointed out that some personal factors of the patients before treatment contributed to the prognosis.

\section{Limitations}

The study had several limitations. First limitation was the lack of blood sample analysis after IVT. It will be valu- able to investigate the short-term or long-term outcomes and their correlation with biomarkers after IVT in stroke patients. The record and analysis of blood biomarkers corresponding to symptoms after therapy may indicate some pathophysiology of the disease. Second, it was a single-center cohort, hence existence of selection bias and relatively small sample size. Third, restricted range of confounding variable examined may lead to chance findings and incomplete adjustment for confounding. Moreover, the use of antiplatelet or anticoagulants may influence the results of platelet-related parameters, but we lack data of this drug-using history in patients. Well-known and repeatedly verified predictors for good outcome were not found like onset-to-treatment time [25], in our study. It could be because our study only had a sample size of 241 and the different definition of good outcome. Finally, there was no external validation in an independent dataset. Further studies in large population to verify the findings need to be conducted.

\section{Conclusions}

The study investigated the baseline characters that influence 3-month clinical outcomes in patients with AIS after thrombolytic therapy. We found that NIHSS, TOAST, DM, and MPV were important predictors for functional outcomes at 3 months by multivariate logistic regression analysis. Lower NIHSS and MPV levels upon admission predicted good outcomes. The correlations between MPV, PDW, and PLR in prognostic assessment in stroke patients were enlightening for further study.

\section{Statement of Ethics}

The study was performed with the appropriate participants' informed consent in compliance with the Helsinki Declaration. Ethical approval was obtained from the Ethics Committee of Nanjing Lishui People's Hospital and Affiliated Zhongda Hospital of Southeast University (reference number: ZDSYLL159-P01).

\section{Conflict of Interest Statement}

The authors declare that they have no competing interests.

\section{Funding Sources}

This work was funded by Nanjing Medical science and technology development general project (YKK18218). 


\section{Author Contributions}

Y.Y. and Y.G. conceived and designed the research. Y.Y., X.C., H.W., and R.Z., performed the study and analyzed the data. S.Z., H.X., and X.G. provided experimental assistance. X.C. and Y.Y. wrote the manuscript.

\section{References}

1 Herpich F, Rincon F. Management of acute ischemic stroke. Crit Care Med. 2020;48(11): 1654-63.

2 Mizuma A, Yenari MA. Clinical perspectives on ischemic stroke. Exp Neurol. 2021;338: 113599.

3 Powers WJ, Rabinstein AA, Ackerson T, Adeoye OM, Bambakidis NC, Becker K, et al. 2018 guidelines for the early management of patients with acute ischemic stroke: a guideline for healthcare professionals from the American Heart Association/American Stroke Association. Stroke. 2018;49(3):e46110.

4 Wang W, Li M, Chen Q, Wang J. Hemorrhagic transformation after tissue plasminogen activator reperfusion therapy for ischemic stroke: mechanisms, models, and biomarkers. Mol Neurobiol. 2015;52(3):1572-9.

5 Guo Y, Yang Y, Zhou M, He L. Risk factors of haemorrhagic transformation for acute ischaemic stroke in Chinese patients receiving intravenous recombinant tissue plasminogen activator: a systematic review and meta-analysis. Stroke Vasc Neurol. 2018;3(4):203-8.

6 Fuentes QE, Fuentes QF, Andrés V, Pello OM, Font de Mora J, Palomo GI. Role of platelets as mediators that link inflammation and thrombosis in atherosclerosis. Platelets. 2013;24(4):255-62.

7 Sadeghi F, Kovács S, Zsóri KS, Csiki Z, Bereczky Z, Shemirani AH. Platelet count and mean volume in acute stroke: a systematic review and meta-analysis. Platelets. $2020 \mathrm{Aug}$ 17;31(6):731-9.

8 Chu SG, Becker RC, Berger PB, Bhatt DL, Eikelboom JW, Konkle B, et al. Mean platelet volume as a predictor of cardiovascular risk: a systematic review and meta-analysis. J Thromb Haemost. 2010;8(1):148-56.

9 Quan W, Chen Z, Yang X, Li J, Li X, Weng Y, et al. Mean platelet volume/platelet count ra-

\section{Data Availability Statement}

All data generated or analyzed during this study are included in this article. tio as a predictor of 90-day outcome in large artery atherosclerosis stroke patients. Int J Neurosci. 2017;127(11):1019-27.

10 Adams HP Jr, Bendixen BH, Kappelle LJ, Biller J, Love BB, Gordon DL, et al. Classification of subtype of acute ischemic stroke. Definitions for use in a multicenter clinical trial. TOAST. Trial of Org 10172 in acute stroke treatment. Stroke. 1993;24(1):35-41.

11 Kolominsky-Rabas PL, Weber M, Gefeller O, Neundoerfer B, Heuschmann PU. Epidemiology of ischemic stroke subtypes according to TOAST criteria: incidence, recurrence, and long-term survival in ischemic stroke subtypes: a population-based study. Stroke. 2001; 32(12):2735-40.

12 Adams HP Jr, Bendixen BH, Kappelle LJ, Biller J, Love BB, Gordon DL, et al. Classification of subtype of acute ischemic stroke. Definitions for use in a multicenter clinical trial. TOAST. Trial of Org 10172 in acute stroke treatment. Stroke. 1993 Jan;24(1):3541.

13 Bakogiannis C, Sachse M, Stamatelopoulos K, Stellos K. Platelet-derived chemokines in inflammation and atherosclerosis. Cytokine. 2019;122:154157.

14 Lordan R, Tsoupras A, Zabetakis I. Platelet activation and prothrombotic mediators at the nexus of inflammation and atherosclerosis: potential role of antiplatelet agents. Blood Rev. 2021;45:100694.

15 Borissoff JI, Spronk HM, ten Cate H. The hemostatic system as a modulator of atherosclerosis. N Engl J Med. 2011;364(18):1746-60.

16 Gawaz M, Langer H, May AE. Platelets in inflammation and atherogenesis. J Clin Invest. 2005;115(12):3378-84.

17 İdil Soylu A, Arıkan Cortcu S, Uzunkaya F, Atalay YO, Bekçi T, Güngör L, et al. The correlation of the platelet-to-lymphocyte ratio with the severity of stenosis and stroke in pa- tients with carotid arterial disease. Vascular. 2017 Jun;25(3):299-306.

18 Horne BD, Anderson JL, John JM, Weaver A, Bair TL, Jensen KR, et al.; Intermountain Heart Collaborative Study Group. Which white blood cell subtypes predict increased cardiovascular risk? J Am Coll Cardiol. 2005 May 17;45(10):1638-43.

19 Kamath S, Blann AD, Lip GY. Platelet activation: assessment and quantification. Eur Heart J. 2001;22(17):1561-71.

20 Thon JN, Italiano JE Jr. Does size matter in platelet production? Blood. 2012;120(8): 1552-61.

21 Liani R, Halvorsen B, Sestili S, Handberg A, Santilli F, Vazzana N, et al. Plasma levels of soluble CD36, platelet activation, inflammation, and oxidative stress are increased in type 2 diabetic patients. Free Radic Biol Med. 2012; 52(8):1318-24

22 Ishikawa T, Shimizu M, Kohara S, Takizawa S, Kitagawa Y, Takagi S. Appearance of WBCplatelet complex in acute ischemic stroke, predominantly in atherothrombotic infarction. J Atheroscler Thromb. 2012;19(5):494501.

23 Franks ZG, Campbell RA, Weyrich AS, Rondina MT. Platelet-leukocyte interactions link inflammatory and thromboembolic events in ischemic stroke. Ann N Y Acad Sci. 2010; 1207:11-7.

24 Xie D, Xiang W, Weng Y, Li J, Xu L, Zhang X, et al. Platelet volume indices for the prognosis of acute ischemic stroke patients with intravenous thrombolysis. Int J Neurosci. 2019; 129(4):344-9.

25 Lees KR, Bluhmki E, von Kummer R, Brott TG, Toni D, Grotta JC, et al. Time to treatment with intravenous alteplase and outcome in stroke: an updated pooled analysis of ECASS, ATLANTIS, NINDS, and EPITHET trials. Lancet. 2010;375(9727):1695-703. 